\title{
Avaliação da atividade de aldeídos aromáticos e suas oximas frente à enzima acetilcolinesterase eritrocitária humana
}

\author{
Maíra Dias Mangueira Bastosa, Victória Laysna dos Anjos Santos ${ }^{\mathrm{a}, \mathrm{b}}$, Arlan de Assis Gonsalves ${ }^{\mathrm{a}, \mathrm{c}}$, Cleônia \\ Roberta Melo Araújo ${ }^{\mathrm{a}, \mathrm{b}^{*}}$ \\ ${ }^{a}$ Grupo de Química Aplicada à Farmácia, Universidade Federal do Vale do São Francisco, Petrolina, 56304-917, Pernambuco, Brasil. \\ *cleonia.araujo@univasf.edu.br \\ b Programa de Pós-Graduação em Ciências da Saúde e Biológicas, Universidade Federal do Vale do São Francisco, Petrolina, 56304-917, Pernambuco, \\ Brasil. \\ c Programa de Pós-Graduação em Ciências dos Materiais, Universidade Federal do Vale do São Francisco, Juazeiro, 48902-300, Bahia, Brasil.
}

Recebido: 11 julho 2017 / Aceito: 8 setembro 2017 / Publicado online: 27 setembro 2017

\begin{abstract}
Resumo
Considerando a importância terapêutica das drogas inibidoras da acetilcolinesterase, bem como dos regeneradores da enzima, em casos de intoxicações por organofosforados. A atividade de aldeídos aromáticos e suas respectivas oximas foi avaliada frente à acetilcolinesterase eritrocitária humana. As oximas foram preparadas a partir da oximação do benzaldeído, do 4hidroxibenzaldeído, do 4-hidroxi-3-metoxibenzaldeído e do 4-dimetialminobenzaldeído, e em seguida, o ensaio de Ellman foi empregado para avaliar o comportamento destes compostos frente à acetilcolinesterase ativa e inibida por diclorvós. Os aldeídos avaliados apresentaram atividade inibidora da acetilcolinesterase nas porcentagens de $21,33 \%, 53,00 \%, 44,00 \%$ e $62,67 \%$ respectivamente para benzaldeído, 4-hidroxibenzaldeído, 4-hidroxi-3-metoxibenzaldeído e 4- dimetilminobenzaldeído. Dentre as oximas avaliadas, apenas a dimetilaminobenzaldoxima apresentou atividade inibitória (36,67\%). A benzaldoxima, a 4 hidroxibenzaldoxima e a 4-hidroxi-3-metoxibenzaldoxima mostraram-se capazes de regenerar modestamente a atividade enzimática em 9,21\%,32,83\% e 8,90\%, respectivamente, quando a enzima se encontrava inibida por diclorvós. Os aldeídos aromáticos testados apresentaram potencial atividade inibitória da acetilcolinesterase eritrocitária, na concentração de $100 \mathrm{mM}$, sendo o maior potencial demonstrado pelo 4-dimetilamibenzaldeído, com 62,67\% de inibição. As oximas, com exceção da dimetilaminobenzaldoxima, se comportaram como regeneradores fracos da enzima inibida por diclorvós, mostrando a relevância do grupo oxima para a regeneração da enzima inibida por organofosforados.
\end{abstract}

Palavras-chave: Organofosforado, diclorvós, pralidoxima, teste de Ellman

\section{Evaluation of the activity of aromatic aldehydes and their oximes against human erythrocyte acetylcholinesterase enzyme}

\begin{abstract}
Considering the therapeutic importance of the acetylcholinesterase inhibitor drugs, as well, enzyme regenerators drugs, in cases of organophosphate poisoning. The activity of aromatic aldehydes and their oximes was evaluated against human erythrocyte acetylcholinesterase. Oximes were prepared from the oximation of benzaldehyde, 4-hydroxybenzaldehyde, 4-hydroxy-3methoxybenzaldehyde and 4-dimethylminobenzaldehyde, and then the Ellman assay was used to evaluate the behavior of these compounds against active acetylcholinesterase and the enzyme inhibited by dichlorvos. The evaluated aldehydes showed acetylcholinesterase inhibitory activity in the percentage of $21.33 \%, 53.00 \%, 44.00 \%$ and $62.67 \%$, respectively, for benzaldehyde, 4-hydroxybenzaldehyde, 4-hydroxy-3-methoxybenzaldehyde and 4- dimethylminobenzaldehyde. Among the oximes evaluated, only dimethylaminobenzaldoxime showed inhibitory activity (36.67\%). Benzaldoxime, 4hydroxybenzaldoxime and 4-hydroxy-3-methoxybenzaldoxime could modestly regenerate the enzymatic activity in $9.21 \%$, $32.83 \%$ and $8.90 \%$, respectively, when the enzyme was inhibited by dichlorvos. The aromatic aldehydes tested showed a potential inhibitory activity of erythrocyte acetylcholinesterase at the concentration of $100 \mathrm{mM}$, the highest potential being demonstrated by 4-dimethylamibenzaldehyde, with $62.67 \%$ inhibition. The oximes, except for dimethylaminobenzaldoxime, behaved as weak regenerators of the enzyme inhibited by dichlorvos, showing the relevance of the oxime group for the regeneration of the enzyme inhibited by organophosphates.
\end{abstract}

Keywords: Organophosphorus, dichlorvos, pralidoxime, Ellman's test 


\section{Introdução}

A acetilcolinesterase (AChE) é a enzima responsável por hidrolisar a acetilcolina ( $\mathrm{ACh}$ ) na fenda sináptica, de forma a finalizar a transmissão neuronal, sendo esta chamada de sinapse colinérgica (Araújo et al., 2016). Inibidores da acetilcolinesterase (IAChE), ou anticolinesterásicos, atuam inibindo a hidrólise da $\mathrm{ACh}$ pela $\mathrm{AChE}$, aumentando assim a amplitude e a duração da ação deste neurotransmissor (Massoud et al., 2011). De acordo com o modo de ação, os inibidores de AChE podem ser divididos em dois grupos: irreversíveis e reversíveis. Os inibidores reversíveis na sua maioria têm aplicações terapêuticas, enquanto que os inibidores irreversíveis são caracterizados por apresentar efeitos tóxicos (Colovic et al., 2013).

Os inibidores reversíveis apresentam grande importância terapêutica no tratamento da miastenia gravis, da doença de Alzheimer, na retenção urinária, bem como, para reverter o bloqueio neuromuscular promovido por miorrelaxantes adespolarizantes (Colovic et al., 2013; Araújo et al., 2016). A neostigmina, o edrofônio, a rivastigmina e a donepezila são exemplos de fármacos IAChE (Mehta et al., 2012).

Como IAChE irreversíveis têm-se os pesticidas organofosforados, que apesar da elevada toxicidade ainda são largamente empregados, atualmente correspondem entre $24,4 \%$ e $33 \%$ dos inseticidas consumidos no Mundo, mas em 1987 chegaram a representar $71 \%$ (Linhares, 2014). Os sintomas típicos de intoxicações por organofosforados são: agitação, fraqueza muscular, fasciculações musculares, miose, hipersalivação, sudorese, insuficiência respiratória, perda de consciência, confusão, convulsões e até a morte (Colovic et al., 2013). Um dos tratamentos para estes casos, envolve o uso de reativadores da $\mathrm{AChE}$, como a Pralidoxima, uma oxima capaz de reverter a inibição da $\mathrm{AChE}$ e assim restaurar a atividade enzimática (Worek et al., 2005).

Considerando a alta gravidade das patologias que podem ser tratadas com o uso de IAChE, bem como, a importância terapêutica da reativação desta enzima em casos de intoxicações por organofosforados, faz-se de grande necessidade a avaliação da ação de substâncias frente à enzima para o desenvolvimento de novas moléculas bioativas. Os ensaios utilizados atualmente para verificar o potencial de inibição ou regeneração da $\mathrm{AChE}$ in vitro, em geral, empregam a enzima obtida de diversas fontes, podendo esta ser isolada de uma espécie de peixe elétrico, Electrophorus electricus (Teleostei, Gymnotiformes), do sangue bovino e de eritrócitos humanos (Rapport et al., 2013).

As enzimas humanas geram resultados mais fiéis, visto que, há diferenças entre a enzima encontrada nas diferentes espécies animais. Contudo, AChE de eritrócitos humanos e $\mathrm{AChE}$ recombinante têm elevados custos para aquisição. $\mathrm{O}$ emprego da AChE presente nos eritrócitos fantasmas livres de hemoglobina (Hemoglobin-free erythrocyte ghosts) é uma alternativa atraente, pois o ensaio é realizado na enzima humana a um baixo custo.

Oliveira-Silva et al. (2000) empregaram o etil-paraoxon para inibir a AChE sanguínea em concentrações variadas $(0,0625 \pm 50,0 \mu \mathrm{g} / \mathrm{mL}$ de sangue). O diclorvós (2,2diclorovinil dimetil fosfato) é um organofosforado, inibidor direto das colinesterases, com amplo uso no ambiente doméstico, no combate a endemias e pragas agrícolas (Sotiropouloua et al., 2005). Contudo, não há estudos de regeneração da AChE humana inibida pelo diclorvós, e este é um atraente organofosforado para realização de testes in vitro, visto que é hidrossolúvel (Linhares, 2014).

A presença de anéis piridínico quaternário e de grupos oximas nas posições 2 e 4 são características importantes para as moléculas capazes de reativar a AChE inibida por organofosforados (Jokanovic e Prostan, 2009). Então, considerando essas características, a atividade dos seguintes aldeídos aromáticos: benzaldeído, 4-hidroxibenzaldeído, 4hidroxi-3-metoxibenzaldeído, 4-dimetialminobenzaldeído e suas oximas foi avaliada frente à enzima $\mathrm{AChE}$ presente na proteína ghost humana não inibida e inibida por diclorvós.

\section{Material e Métodos}

Todos os solventes e reagentes, de pureza analítica, foram utilizados sem purificação adicional. Água deionizada foi empregada nos ensaios de avaliação da inibição e reativação da AChE eritrocitária. $\mathrm{O}$ andamento das reações foi acompanhado por cromatografia em camada delgada analítica (CCDA) utilizando folhas de alumínio recobertas de sílica gel 60 (70-230 mesh) com indicador de fluorescência $U^{254}$. As visualizações dos compostos foram realizadas usando luz ultravioleta e iodo como reveladores. As purificações por cromatografia em coluna foram realizadas utilizando sílica gel 60 (70-230 mesh) da Aldrich ${ }^{\circledR}$.

\section{Síntese da oximas aromáticas}

A benzaldoxima, a 4-hidroxibenzaldoxima, a 4-hidroxi-3metoxi-benzaldoxima e a 4-dimetilaminobenzaldoxima foram preparadas a partir da oximação do benzaldeído, do 4hidroxibenzaldeído, do 4-hidroxi-3-metoxibenzaldeído e do 4-dimetialminobenzaldeído, respectivamente, utilizando cloridrato de hidroxilamina em meio básico (Araújo e Gonsalves, 2015; Figura 1).

A síntese da benzaldoxima, da 4-hidroxibenzaldoxima (Ponto de Fusão - PF: $121-125^{\circ} \mathrm{C}$; lit. $112^{\circ} \mathrm{C}$; Brady e Dunn, 1914), da 4-hidroxi-3metoxi-benzaldoxima (PF: $119-121{ }^{\circ} \mathrm{C}$; lit.117,5-119 ${ }^{\circ} \mathrm{C}$; Bodil e Sine, 1991) e da 4dimetilaminobenzaldoxima (PF: $140-141{ }^{\circ} \mathrm{C}$; lit. 141,5-143 ${ }^{\circ} \mathrm{C}$; Field et al., 1961) foram confirmadas mediante determinação do ponto de fusão $(\mathrm{PF})$ e posterior comparação com dados disponíveis na literatura científica.

\section{Avaliação da atividade frente à AChE eritrocitária humana}

A pesquisa atendeu os princípios éticos e obedeceu a Resolução 466/12/CNS/MS, que regulamenta a pesquisa envolvendo seres humanos. Sendo aprovada pelo Comitê de Ética e Deontologia em Estudos e Pesquisa, da Universidade Federal do Vale do São Francisco com protocolo número 0003/111214.

Para realizar os ensaios de avaliação da atividade dos aldeídos e suas oximas frente à $\mathrm{AChE}$ foi necessário o isolamento adequado da proteína ghost sem resquícios de interferentes que pudessem prejudicar a conformidade dos resultados, como a presença de plasma e hemoglobina. 
<smiles>[R]c1ccc(C=O)cc1[R2]</smiles>

$$
\begin{array}{lll}
\mathrm{R}_{1}-\mathrm{H} & \text { (1) } & \mathrm{R}_{1}-\mathrm{OH} \\
\mathrm{R}_{2}-\mathrm{H} & & \mathrm{R}_{2}-\mathrm{OMe} \\
& & \mathrm{R}_{1}-\mathrm{N}(\mathrm{Me})_{2} \\
\mathrm{R}_{1}-\mathrm{OH} & \text { (2) } & \mathrm{R}_{2}-\mathrm{H} \\
\mathrm{R}_{2}-\mathrm{H} & &
\end{array}
$$

$$
\begin{aligned}
& \mathrm{R}_{1}-\mathrm{H} \\
& \mathrm{R}_{2}-\mathrm{H} \\
& \text { (5) } \begin{array}{l}
\mathrm{R}_{1}-\mathrm{OH} \\
\mathrm{R}_{2}-\mathrm{OMe}
\end{array} \\
& \mathrm{R}_{1}-\mathrm{OH} \\
& \mathrm{R}_{2}-\mathrm{H} \\
& \text { (6) } \begin{array}{l}
\mathrm{R}_{1}-\mathrm{N}(\mathrm{Me})_{2} \\
\mathrm{R}_{2}-\mathrm{H}
\end{array}
\end{aligned}
$$

Figura 1. Esquema reacional de obtenção da benzaldoxima (5), da 4-hidroxibenzaldoxima (6), da 4-hidroxi-3-metoxibenzaldoxima (7) e da 4-dimetilaminobenzaldoxima (8) a partir do benzaldeído (1), do 4-hidroxibenzaldeído (2), do 4-hidroxi-3-metoxibenzaldeído (3) e do 4dimetialminobenzaldeído (4), respectivamente.

Assim, o plasma sanguíneo foi separado do concentrado de hemácias para que não houvesse a obtenção conjunta da butirilcolinesterase (BChE), enzima que apresenta diferença estrutural do sítio catalítico quando comparada com a $\mathrm{AChE}$ eritrocitária, influenciando na acomodação e seletividade dos diferentes inibidores da AChE (Freitas et al., 2009). A hemoglobina possui a absorbância no mesmo comprimento de onda $(410 \mathrm{~nm})$ que o ácido tionitrobenzoico, composto responsável por quantificar a atividade enzimática da $\mathrm{AChE}$ no método de Ellman (Araujo et al., 2016). Assim, esta foi liberada após a lise das hemácias e removida por meio de lavagens sucessivas com tampão (Iménez-Díaz e MartinezMonge, 2000).

Obtenção da AChE presente na proteína ghost não inibida e inibida por Diclorvós

As amostras de sangue humano foram aliquotadas em tubos tipo falcon em volumes de 2,00 mL. As hemácias foram separadas do plasma por meio de centrifugação a $5000 \mathrm{rpm}$ por 15 minutos. O plasma foi descartado e aos tubos foram adicionados $4,50 \mathrm{~mL}$ de tampão de lise $\left(\mathrm{NaH}_{2} \mathrm{PO}_{4} / \mathrm{Na}_{2} \mathrm{HPO}_{4}\right.$ $20,00 \mathrm{mM}$ - pH 7.60). Após adição do tampão os tubos foram congelados a $-6{ }^{\circ} \mathrm{C}$ e mantidos nesta temperatura por 24 horas (Oliveira-Silva et al., 2000).

As misturas foram descongeladas, as hemácias foram homogeneizadas e submetidas a três centrifugações a 5000 rpm durante 15 minutos cada. Ao final de cada processo o sobrenadante foi desprezado em hipoclorito de sódio e as hemácias ressuspensas em tampão de lise. Após a terceira centrifugação, foi retirado o sobrenadante e dividiu-se o sedimento em duas alíquotas, a uma delas adicionou-se 500,00 $\mu \mathrm{L}$ de tampão de análise $\left(\mathrm{NaH}_{2} \mathrm{PO}_{4} / \mathrm{Na}_{2} \mathrm{HPO}_{4} 120,00 \mathrm{mM}\right.$ - $\mathrm{pH}$ 7.60) obtendo-se a enzima ativa (não inibida). À outra amostra acrescentou-se $3,00 \mathrm{~mL}$ da solução inibidora de diclorvós $\left(1,00 \mathrm{mg} \mathrm{mL}^{-1}\right)$, deixando agir por 15 minutos. Em seguida, a amostra foi submetida a duas centrifugações (5000 rpm por 15 minutos), sendo que o sobrenadante era descartado ao fim de cada uma delas e adicionado $4,50 \mathrm{~mL}$ do tampão de análise. Por fim, ao retirar o último sobrenadante, adicionouse 500,00 $\mu \mathrm{L}$ de tampão de análise e obteve-se a (Hemoglobinfree erythrocyte ghosts) proteína ghost inibida (Oliveira-Silva et al., 2000).

Avaliação da atividade enzimática da AChE presente na proteína ghost e proteína ghost inibida por Diclorvós

Em um tubo de ensaio foram adicionados $25,00 \mu \mathrm{L}$ de proteína ghost não inibida e $75,00 \mu \mathrm{L}$ de solução $10 \%$ $\mathrm{C}_{2} \mathrm{H}_{6} \mathrm{O} / \mathrm{H}_{2} \mathrm{O}$. Transcorridos 30 minutos foram adicionados aos tubos 2,00 mL de solução tampão de análise, $0,50 \mathrm{~mL}$ de solução de 5,5'-ditiobis (2-nitrobenzóico) (DTNB) e 0,50 mL de solução de iodeto de acetiltiocolina (IAChT). Ao finalizar a adição dos reagentes a cada tubo, as alíquotas foram analisadas cineticamente a $412 \mathrm{~nm}$ por 3,5 minutos em espectrofotômetro. $\mathrm{O}$ mesmo procedimento foi realizado para determinar a atividade catalítica da AChE na proteína ghost inibida. Este procedimento foi realizado em triplicata.

\section{Avaliação quantitativa do potencial das substâncias em inibir a AChE}

Em um tubo de ensaio foi adicionado $25,00 \mu \mathrm{L}$ de proteína ghost não inibida e $75,00 \mu \mathrm{L}$ de solução $10 \% \mathrm{C}_{2} \mathrm{H}_{6} \mathrm{O} / \mathrm{H}_{2} \mathrm{O}$ substância testada na concentração de $100 \mathrm{mM}$. Transcorridos 30 minutos foram adicionados aos tubos $2,00 \mathrm{~mL}$ de solução tampão de análise, $0,50 \mathrm{~mL}$ de solução de DTNB e $0,50 \mathrm{~mL}$ de solução de IAChT. Ao finalizar a adição dos reagentes a cada tubo, as alíquotas foram analisadas cineticamente a 412 $\mathrm{nm}$ por 3,5 minutos. Este procedimento foi realizado em triplicata.

Avaliação quantitativa do potencial das substâncias em reativar a AChE inibida por Diclorvós

Para avaliar a capacidade reativadora das substâncias testadas, empregou-se a mesma metodologia descrita 
anteriormente, porém utilizando a enzima previamente inibida por diclorvós. Desta forma, em um tubo de ensaio adicionouse $25,00 \mu \mathrm{L}$ de proteína ghost inibida e $75,00 \mu \mathrm{L}$ de solução $10 \% \mathrm{C}_{2} \mathrm{H}_{6} \mathrm{O} / \mathrm{H}_{2} \mathrm{O}$ substância testada na concentração de 100 $\mathrm{mM}$. Transcorridos 30 minutos foram adicionados aos tubos 2,00 mL de solução tampão de análise, $0,50 \mathrm{~mL}$ de solução de DTNB e $0,50 \mathrm{~mL}$ de solução de IAChT. Ao finalizar a adição dos reagentes a cada tubo, as alíquotas foram analisadas cineticamente a $412 \mathrm{~nm}$ por 3,5 minutos. Este procedimento foi realizado em triplicata.

\section{Determinação da atividade catalítica da AChE}

A atividade catalítica da AChE foi calculada a partir da inclinação (coeficiente angular) da região linear da curva de cinética obtida por espectrofotometria de absorção molecular, a qual corresponde à variação de absorbância no decorrer do tempo. A equação 1 foi empregada para quantificar a atividade enzimática da proteína ghost, utilizando uma equação descrita por Razzino (2007; Tabela 1), sendo expressa em U de enzima ( $1 \mathrm{U}$ de enzima hidrolisa um $\mu \mathrm{mol}$ de substrato por minuto). As equações para quantificação dos percentuais de inibição e regeneração da AChE também estão descritas na Tabela 1.

Tabela 1. Equações utilizadas para os cálculos da atividade enzimática e percentuais de inibição e regeneração da acetilcolinesterase.

\begin{tabular}{lc}
\hline \multicolumn{1}{c}{ Variável } & \multicolumn{1}{c}{ Equação } \\
\hline Atividade catalítica & $(\Delta \mathrm{A} / \Delta \mathrm{T} \times 1000) \times[\mathrm{FD} /(\varepsilon \times \mathrm{xd})]$ \\
enzimática $(\mathrm{Z})$ & $\left(\mathrm{Z}_{\mathrm{L}}-\mathrm{Z}_{\mathrm{T}}\right) / \mathrm{Z}_{\mathrm{L}} \times 100$ \\
$\%$ inibição & $\left(\mathrm{Z}_{\mathrm{R}}-\mathrm{Z}_{\mathrm{I}}\right) / \mathrm{Z}_{\mathrm{L}} \times 100$ \\
\hline
\end{tabular}

Legenda: $\Delta \mathrm{A}$ : variação de absorbância; $\varepsilon$ : coeficiente de absorção do ânion 5-tio-2-nitrobenzoato $\left(1,36 \mathrm{mmol}^{-1} \mathrm{~mm}^{-1}\right)$; d: o caminho ótico $(\mathrm{mm}) ; \Delta \mathrm{t}$ : variação do tempo (minutos); FD: fator de diluição da amostra de "proteína ghost" $\left(\mathrm{v} \mathrm{v}^{-1}\right)$. Atividade catalítica enzimática $(A C E)$ da $A C h E$ na proteína "ghost" inibida $\left(Z_{I}\right)$ e não inibida $\left(Z_{L}\right)$; $\mathrm{ACE}$ da $\mathrm{AChE}$ proteína "ghost" exposta à substância inibidora $\left(\mathrm{Z}_{\mathrm{T}}\right) \mathrm{e}$ reativadora $\left(Z_{R}\right)$ testadas.

\section{Resultados e Discussão}

Nos ensaios foram testados tantos os aldeídos precursores, o benzaldeído, o 4-hidroxibenzaldeído, o 4-hidroxi-3metoxibenzaldeído e o 4-dimetialminobenzaldeído, quanto as oximas sintéticas, todos na concentração de $100 \mathrm{mM}$ em soluções $10 \% \mathrm{C}_{2} \mathrm{H}_{6} \mathrm{O} / \mathrm{H}_{2} \mathrm{O}$.

A 4-dimetilaminobenzaldoxima e todos os aldeídos investigados atuaram na enzima de forma a inibi-la, sendo a inibição de $36,67 \%$ para a 4-dimetilaminobenzaldoxima, e de $21,33 \%, 53,00 \%, 44,00 \%$ e $62,67 \%$, respectivamente para o benzaldeído, o 4-hidroxibenzaldeído, o 4-hidroxi-3metoxibenzaldeído e o 4-dimetialminobenzaldeído, (Tabela 2). Deste modo foi possível observar que dentre os aldeídos aromáticos testados os que se apresentam substituídos com grupos doadores de elétrons, o grupamento hidroxila e dimetilamina, na posição para mostram-se como inibidores mais potentes da AChE eritrocitária. Li e colaboradores (2016) também observaram que dentre os inibidores testados em seu estudo, os que apresentaram melhor atividade inibitória frente a $\mathrm{AChE}$ foram os que o anel aromático presente em seus derivados sintéticos apresentavam-se substituídos na posição para, ressaltando assim a importância de substituintes nesta posição para a atividade inibitória.

Tabela 2. Porcentagem de inibição ( $\% \pm$ Desvio padrão) da AChE eritrocitária na concentração de $100 \mathrm{mM}$.

\begin{tabular}{c|c}
\hline Substância e estrutura Química & Inibição \\
\hline Benzaldeído & $21,33 \pm 3,51$ \\
\hline 4-hidroxi-3-metoxibenzaldeído & \\
\hline 4-dimetilaminobenzaldoxima & $53,00 \pm 3,00$ \\
\hline
\end{tabular}

A benzaldoxima, a 4-hidroxibenzaldoxima e a 4-hidroxi3-metoxibenzaldoxima, na concentração investigada, foram capazes de regenerar 9,21\%,32,83\% e 8,90\% da atividade da AChE eritrocitária inibida por diclorvós, respectivamente (Tabela 3).

Os estudos de relação estrutura-atividade de reativadores da AChE inibida por organofosforados, demonstram que existem quatro fatores estruturais importantes que influenciam na reativação desta enzima, sendo eles: a presença de um nitrogênio quaternário, a estrutura da cadeia que liga dois anéis piridínicos, a presença do grupo oxima e sua posição no anel piridínico (Kucaa et al., 2006; Kassa et al., 2015).

O grupo oxima atua como reativador da AChE quando esta encontra-se inibida por organofosforados, através do ataque nucleofílico ao átomo de fósforo do resíduo de serina fosforilado da $\mathrm{AChE}$, resultando na remoção do grupo fosforil e na consequente reativação da enzima inibida (Korabecnya et al., 2014; Araújo et al., 2016). Este processo depende da nucleofilicidade e orientação do grupo oxima na molécula do 
reativador, assim como da força das interações intermoleculares formadas entre a enzima e o organofosforado, de modo que sua eficácia pode variar de acordo com o organofosforado utilizado (Costa et al., 2011; Kassa et al., 2015).

Tabela 3. Porcentagem de regeneração $(\% \pm$ Desvio padrão) da AChE eritrocitária inibida por diclorvós na concentração de $100 \mathrm{mM}$.

\begin{tabular}{c|c}
\hline Substância e estrutura química & Regeneração \\
\hline Benzaldoxima & $9,21 \pm 1,66$ \\
\hline
\end{tabular}

a Porcentagem de regeneração da AChE inibida (\% \pm DP) $(10 \mathrm{mM})$ e com tempo de atuação de 15 minutos.

No presente estudo foi possível observar a importância do grupo oxima para a reativação da $\mathrm{AChE}$ inibida por organofosforado, uma vez que a oximação dos aldeídos aromáticos permitiu que estas moléculas deixassem de ser inibidores e adquirissem a capacidade de regenerar a atividade enzimática da AChE eritrocitária inibida por diclorvós.

A pralidoxima é o fármaco reativador da $\mathrm{AChE}$ inibida presente na versão atual da Relação Nacional de Medicamentos Essenciais (RENAME), a edição de 2014 (Rename, 2017). Este foi capaz de regenerar $100 \%$ da $\mathrm{AChE}$ eritrocitária inibida por diclorvós na concentração de $10 \mathrm{mM}$ e atuando por 15 minutos na enzima inibida. Apesar da benzaldoxima, da 4-hidroxibenzaldoxima e da 4-hidroxi-3metoxibenzaldoxima serem capazes de regenerar a enzima, esta atividade é baixa quando comparada com a pralidoxima.

A principal diferença estrutural entre a pralidoxima e as oximas testadas é a presença do anel piridínico presente na pralidoxima, assim este resultado corrobora com os estudos que mostram que o anel pirídinico é importante para as moléculas reativadoras da AChE inibida (Worek et al., 2012;
Chambers et al., 2016). A influência dos substituintes aromáticos das oximas testadas pode ser analisado através da comparação entre as atividades destas sobre a AChE. Ao comparar o potencial de reativação da benzaldoxima $(9,21 \pm 1,66)$ e do 4-hidroxibenzaldoxima $(32,83 \pm 1,73)$ é notado que a substituição do anel aromático na posição para pelo grupo hidroxila potencializou a ação da molécula. Contudo, ao introduzir além da hidroxila uma metoxila na posição meta esse efeito foi eliminado, como ocorreu com 4hidroxi-3-metoxibenzaldoxima $(8,90 \pm 0,25)$ que apresentou atividade semelhante ao seu análogo simplificado, a benzaldoxima $(9,21 \pm 1,66)$. Além disso, observou-se também que a oxima aromática quando substituída na posição para pelo grupo dimetilamina, a 4-dimetilaminobenzaldoxima, não foi capaz de reativar a $\mathrm{AChE}$ eritrocitária inibida por diclorvós, apresentando atividade inibidora da enzima.

\section{Conclusões}

A semelhança estrutural apresentada entre os compostos testados e a pralidoxima se mostrou importante para a capacidade dos compostos atuarem na enzima AChE. Tanto que, os aldeídos foram capazes de inibir a $\mathrm{AChE}$ eritrocitária humana de forma significativa, na concentração de $100 \mathrm{mM}$, sendo o maior potencial demonstrado pelo 4dimetilamibenzaldeído, com $62,67 \%$ de inibição. A benzaldoxima, a 4-hidroxibenzaldoxima e a 4-hidroxi-3metoxibenzaldoxima se comportaram como regeneradores fracos da enzima inibida por diclorvós, este resultado mostra a relevância do grupo oxima, bem como, do anel piridínico para a regeneração da $\mathrm{AChE}$ inibida por organofosforados.

\section{Agradecimentos}

Ao $\mathrm{CNPq}$, à Fundação de Amparo à Pesquisa de Pernambuco (FACEPE) e à Universidade Federal do Vale do São Francisco (UNIVASF).

\section{Referências}

Araújo, C. R. M.; Gonsalves, A. A. 2015. Oximas: propriedades químicas, métodos de preparação e aplicações na síntese de grupos funcionais nitrogenados. Revista Virtual de Química, 7(4):1469-1495.

Araújo, C. R. M.; Santos, V. L. dos A.; Gonsalves A. A. 2016. Acetilcolinesterase - AChE: Uma Enzima de Interesse Farmacológico. Revista Virtual de Química, 8(6): 1818-1834.

Bodil, J.; Sine, L. 1991. Hydrogen bonding and stereochemistry of ringhydroxylated aromatic aldehyde oximes. Crystal structures of three 4hydroxy-substituted benzaldehyde oxime. Acta Chemica Scandinavica, 45: 285-291.

Brady, O. L.; Dunn, F. P. 1914. LXXXIV. The isomerism of the oximes. Part III. The hydroxybenzaldoximes. Journal of Chemical Society, PerkinTransaction, 105:821-830.

Chambers, J. E.; Chambers, H. W.; Funck, K. E.; Meek, E. C.; Pringle, R. B.; Ross, M. K. 2016. Efficacy of novel phenoxyalkyl pyridinium oximes as brainpenetrating reactivators of cholinesterase inhibited by surrogates of sarin and VX. Chemico-Biological Interactions, 259(2016):154-159.

Colovic, M. B.; Krstić D. Z.; Lazarević-Pašti, T. D.; Bondžić, A. M.; Vasić, V. M. 2013 Acetylcholinesterase Inhibitors: Pharmacology and Toxicology. Current. Neuropharmacology, 11(3): 315-335.

Costa, M. D.; Freitas, M. L.; Soares, F. A. A.; Santana Carratu, V. S.; Brandão, R. 2011. Potential of two new oximes in reactivate human acetylcholinesterase and butyrylcholinesterase inhibited by organophosphate compounds: An in vitro study. Toxicology in vitro, 25 (2011): 2120-2123.

Field, L.; Hughmark, P. B.; Shumaker, S. H. W.; Marshall, S. 1961. 
Isomerization of Aldoximes to Amides under Substantially Neutral Conditions. Journal of the American Chemical Society, 83(8): 19831987.

Freitas, H. F.; Paz, O. S.; Castilho, M. S. 2009. Estudos de QSAR 3D para um conjunto de inibidores de butirilcolinesterase humana. Química Nova, 32(8): 2114-2121.

Iménez-Díaz, M.; Martinez-Monge, V. 2000. Validación de la determinación de colinesterasa plasmática humana a $340 \mathrm{nM}$. Revista Biomédica, 11(2):91-98.

Jokanovic, M.; Prostan, M. 2009. Pyridinium oximes as cholinesterase reactivators. Structure-activity relationship and efficacy in the treatment of poisoning with organophosphorus compounds. Current Medicinal Chemistry, 16(17): 2177-2188.

Kassa, J.; Sepsova, V.; Horova, A.; Musilek, K. 2015. A comparison of the reactivating and therapeutic efficacy of two novel bispyridinium oximes (K920, K923) with the oxime K203 and trimedoxime in tabun-poisoned rats and mice. Journal of applied biomedicine, 13 (2015): 299-304.

Korabecnya, J.; Soukupb, O.; Dolezalb, R.; Spilovskaa, K.; Nepovimovaa, E.; Andrsa, M.; Nguyenc, T. D.; Juna, D.; Musileka, K.; KucerovaChlupacovac, M.; Kucab, K. 2014. From Pyridinium-based to Centrally Active Acetylcholinesterase Reactivators. Mini-Reviews in Medicinal Chemistry, 14: 215-221.

Kucaa, K.; Juna, D.; Musilekb, K. 2006. Structural Requirements of Acetylcholinesterase Reactivators. Mini-Reviews in Medicinal Chemistry, 6: 269-277.

Li, J-C.; Zhang, J.; Rodrigues, M. C.; Ding, D-J.; Longo, J. P. F.; Azevedo, R. B.; Muehlmann, L. A.; Jiang, C-S. 2016. Synthesis and evaluation of novel 1,2,3-triazole-based, acetylcholinesterase inhibitors with neuroprotective activity. Bioorganic \& Medicinal Chemistry Letters, 26 (2016): 3881-3885.

Linhares, A. G. 2014. Efeito de pesticidas organofosforados e carbamatos sobre a acetilcolinesterase eritrocitária humana e seu potencial uso como biomarcardor da exposição ocupacional, Pernambuco, Brasil. Dissertação de Mestrado, Pós-Graduação em Ciências Biológicas Universidade Federal de Pernambuco, Recife, Pernambuco. 81 p.

Massoud, F.; Desmarais, J. E.; Gauthier, S. 2011. Switching cholinesterase inhibitors in older adults whit dementia. International Psychogeriatrics, 23(3):372-378.

Mehta, M.; Adem, A.; Sabbagh, M. 2012. New Acetylcholinesterase Inhibitors for Alzheimer's Disease. International Journal of Alzheimer's Disease, 2012: 1-8.

Oliveira-Silva, J. J.; Alves, S. R, Inacio, A. F.; Meyer, A.; Sarcinelli, P. N.; Mattos, R. C.; Ferreira, M. F.; Cunha, J. C.; Moreira, J. C. 2000. Cholinesterase activities determination in frozen blood samples: an improvement to the occupational monitoring in developing countries. Human \& Experimental Toxicology, 19(3):173-177.

Rapport, D. J.; Gaudet, C. L.; Calow, P. 2013. Evaluating and Monitoring the Health of Large-Scale Ecosystems. Ed. Springer Science \& Business Media, Canada, 454p.

Razzino, C. A. 2007. Desenvolvimento, caracterização e utilização de um nanobiossensor enzimático para a determinação de carbaril, São Paulo, Brasil. Dissertação de Mestrado/Universidade de São Paulo. Instituto de Química de São Carlos, São Carlos, São Paulo. 79p.

Rename - Relação Nacional de Medicamentos Essenciais, 2017. Disponível em: <http://portalsaude.saude.gov.br/index.php/oministerio/principal/leia-mais-o-ministerio/471-sctie-raiz/dafraiz/daf/13-daf/18892-teste-versoes-rename>. Acesso em 10 Jul. 2017.

Sotiropouloua, S.; Fournierb, D.; Chaniotakisa, N. A. 2005. Genetically engineered acetylcholinesterase-based biosensor for attomolar detection of dichlorvos. Biosensors and Bioelectronic, 20(11): 2347-2352.

Worek, F.; Reiter G.; Eyer, P, Szinicz. L. 2005. Reactivation kinetics of acetylcholinesterase from different species inhibited by highly toxic organophosphates. Archives of Toxicology, 76(9):523-529.

Worek, F.; Wille, T.; Koller, M.; Thiermann, H. 2012. Reactivation kinetics of a series of related bispyridinium oximes with organophosphateinhibited human acetylcholinesterase structure-activity relationships. Biochemical Pharmacology 83 (2012): 1700-1706. 